\title{
War is a cabaret. Reflejos de la Alemania de entreguerras desde la literatura hasta el cine musical
}

\author{
Isabel CASTELls MolinA \\ Departamento de Filología Española \\ Universidad de La Laguna \\ icastell@ull.edu.es
}

\begin{abstract}
RESUMEN
Este trabajo pretende ilustrar cómo Cabaret, de Bob Fosse, refleja el ambiente de Alemania durante el avance del nazismo, partiendo del Adiós a Berlín, de Cristopher Isherwood. Para ello, analiza la función diegética de los números musicales como microcosmos que recrean en abismo la sociedad que los contempla. Asimismo, veremos cómo este planteamiento realista del cine musical contribuye a la renovación del propio género.
\end{abstract}

Palabras clave: Cabaret, Bob Fosse, Adiós a Berlín, Cristopher Isherwood, cine musical, nazismo, estructura en abismo.

War is a cabaret. Reflections of the German interwar period from literature to musical cinema

\begin{abstract}
This paper aims to show how Bob Fosse's Cabaret reflects the atmosphere in Germany during the advance of Nazism, from Cristopher Isherwood's book Good bye to Berlin. For this purpose, it analyzes the diegetic function of musical numbers conceived as microcosms re-creating in abyme the society who watches them. Furthermore, we will see how this realistic treatment of the musical cinema contributes to the renovation of the genre itself.
\end{abstract}

Keywords: Cabaret, Bob Fosse, Good bye to Berlin, Cristopher Isherwood, musical cinema, Nazism, mise en abyme. 
Si ya resulta cuestionable cualquier pretensión de encontrar un supuesto rigor histórico tanto en el relato literario como en el fílmico, nuestra intención de mostrar el modo en el que una película adscrita al género musical se convierte en reflejo de un complejo y bien conocido momento de la sociedad alemana puede resultar, de antemano, una tarea si no inútil, sí paradójica. Sin embargo, en las páginas que siguen nos proponemos ilustrar cómo, a través fundamentalmente de las canciones y coreografías desarrolladas en el microcosmos de un cabaret berlinés, Bob Fosse logra realizar un retrato de la sociedad alemana de entreguerras que se nos antoja, si cabe, más nítido que el que nos ofrecen los manuales históricos, contagiados muy a menudo de la parcialidad y los recursos de cualquier relato ficticio, tal y como ha establecido Hayden White (2010).

La película Cabaret (1972) se erige desde el principio como un entramado de discursos que Bob Fosse explota a la perfección en una propuesta que, justamente por eso, plantea en primer lugar una revisión del propio género al que pertenece, como veremos pronto.

La película se nutre, según la conocida terminología de Génette (1989a), de tres hipotextos. El primero y principal es el libro de relatos de Cristopher Isherwood Adiós a Berlín (1938), del que nacieron a su vez dos trasvases ${ }^{1}$ al mundo de la escena: por un lado, la obra I am a camera, de Jon Van Durten (1951) - que a su vez dio lugar a una película homónima, de Henry Cornelius (1955)- y, por otro y muy especialmente, el musical de Jon Masteroff estrenado en Broadway en 1951 que da título a nuestra película y que aún hoy se sigue representando, sin ir más lejos, en las salas de Madrid ${ }^{2}$.

Ya desde el inicio del primer relato de su libro («Diario de Berlín. Otoño de 1930»), Isherwood parece estar invitando al lector a percibir lo narrado en clave cinematográfica:

Soy una cámara con el obturador abierto, totalmente pasiva, que registra sin pensar. Registra al hombre que se afeita en la ventana de enfrente y a la mujer del kimono lavándose el cabello. Algún día, habrá que revelar, hacer copias cuidadosamente y fijar todo esto. (Isherwood 2014: 9)

La nada gratuita identificación del narrador autobiográfico con la herramienta básica del cine y de la fotografía -que dio lugar, como vemos, a la obra de Van Durten mencionada más arriba - nos indica que el relato posee una clara intención de erigirse en retrato fidedigno de unos personajes y una época. Veamos ahora, centrán-

${ }^{1}$ Hablamos, con la terminología de Sánchez Noriega (2000: 23-24), de «trasvases» y no de «adaptación», porque el primigenio texto de Isherwood realiza un viaje muy interesante y complejo entre diferentes géneros y lenguajes de contextos culturales variados.

${ }^{2}$ En efecto, en el momento de redacción de estas páginas (mayo de 2016), el musical Cabaret, inspirado en su homónimo de Broadway, se está representando en el teatro Rialto de Madrid, bajo la dirección de Jaime Azpilicueta, y realizará una gira por toda España. Protagonizado por Cristina Castaño y Edu Soto, no tiene, en nuestra opinión, la fuerza ni del original de Masteroff ni de la película de Fosse. 
donos ya exclusivamente en la película de Fosse, cómo dicha pretensión puede encajar en un género cinematográfico como el musical.

Decíamos al principio que Cabaret constituye, entre otras muchas cosas que iremos viendo, una revisión del propio género en el que se inscribe. $\mathrm{Si}$, en efecto, según su definición canónica, el musical se basa en

historias optimistas y de cierta frivolidad, en las que una trama y unos personajes muy simples sirven de soporte para números musicales espectaculares [...] con delicada puesta en escena -vestuario y decorado lujosos, utilización de efectos especiales, cuidada planificación- y recursos más o menos hábiles para vencer la inverosímil situación de que un personaje diga sus diálogos cantando. (Sánchez Noriega 2006: 169),

resultaría, como apuntábamos al principio, imposible pretender que una película de esas características pudiera retomar el propósito eminentemente realista del hipotexto de base. Será, pues, el talento del director el que realice esta pirueta en apariencia imposible y logre no solo contribuir a la redefinición del propio género musical sino, recrear, justamente a través de ella, el universo de Isherwood y la Alemania a la que este nos remite. Como bien afirma Max Cousins, Bob Fosse

abrazó de lleno las nuevas tendencias cinematográficas, de ahí que Cabaret [...] sorprendiese por su modernidad. [...] las canciones de la película constituyen una celebración tan desbordante como amoral del dinero, la vida y el inconformismo sexual tan propio de finales de los años sesenta. Los diferentes números del musical, en los que se entrecruzan los bajos mundos y las atrocidades de los nazis, y los cambios bruscos de momentos de diversión a escenas sombrías, como el canto del himno nazi, estaban ya en la obra original, pero Fosse los supo llevar a la pantalla con indudable maestría. (Cousins 2005: 345-348)

Son varias las ideas que nos interesan de esta cita. Por un lado, Cousins subraya la evidente modernidad de una película que aún hoy mantiene tanto su crudeza como su frescura. En segundo término, establece una sugestiva relación entre el momento histórico recreado en la película y el de su filmación, que permitiría reflexionar sobre el carácter cíclico de la Historia y el modo en el cine permite la catarsis de un multiforme espectro de espectadores de distintos tiempos y contextos ${ }^{3}$.

Pero es la tercera de las ideas la que nos interesa ahora especialmente: la que pone el acento en la «maestría» con la que Fosse se sirve de los números musicales a la hora de convertirlos en contrapuntos a un tiempo lúdicos y escalofriantes de un nazismo en ciernes. En efecto, Cousins habla de mundos que «se entrecruzan» y de «cambios bruscos» que van de la diversión al horror y es precisamente en este difí-

${ }^{3}$ En este sentido, resulta muy significativo que una serie de televisión que aborda el tema de la transexualidad, Transparent (Jill Soloway, 2014), acuda también al Berlín anterior al nazismo para ilustrar el mismo ambiente de libertad sexual que nos muestra Fosse, a través de continuos flash-backs que reconstruyen la vida de los antepasados del protagonista principal. De este modo, igual que ocurre en Cabaret, el hecho de mostrar un momento histórico determinado permite iluminar con más claridad el presente. 
cil pulso de tonos y lenguajes donde reside el principal interés de la película y donde podemos encontrar el propósito de reflejar una realidad histórica tal y como se planteaba Isherwood en las palabras reproducidas más arriba.

Para llevar a cabo esta intención de despojar a la película del tono frívolo propio del cine musical -tono obtenido, como hemos visto, al disociar un argumento endeble de unos números musicales intercalados casi al azar- Bob Fosse utiliza dos procedimientos: por un lado, el de dotar a todas y cada una de las canciones de una función diegética y, por otro, el de ensamblarlas a la trama principal a través de una eficaz utilización del montaje paralelo o contraposición de escenas (Roda 1994: 36). Con este doble recurso, el realizador logra que su película trascienda, como venimos diciendo, los principales rasgos del género:

En Cabaret se iban a tomar tan seriamente la lógica musical que aún estando tan cerca del terreno de la canción y el baile, la película no parecería un musical. Incluso los números de cabaret iban a ser mucho más que meros entretenimientos. Estarían relacionados con la acción dramática, en un fascinante concepto que era debido a Fosse. (Gottfriet 2006: 285)

En este sentido, el espacio aparentemente irreal del escenario del Kit Kat, el cabaret berlinés en el que se desarrolla la trama, consigue el paradójico resultado de que «la ley del realismo» sea «una de las claves de la película», ya que, «como pasaba con las canciones, no había ningún baile donde no se hubiese bailado en la vida real» (Gottfriet 2006: 297). Así las cosas, y considerando que las canciones y coreografías apuntalan y en ocasiones adelantan acontecimientos de la trama, convirtiéndose en parte imprescindible del guion, el escenario del cabaret, lejos de ser un espacio de evasión, se convierte en un reflejo de la sociedad que lo crea y contempla, en una interesante y compleja estructura especular o en abismo (Dällenbach 1991) que duplica las funciones de espectador, personaje y narrador. El viejo tópico del teatrum mundi, sobre el que volveremos al final de estas páginas, se pone al servicio de este singular experimento en el que metaficción y realismo logran una lúcida y a la vez lúdica revisión tanto de la historia como del propio lenguaje cinematográfico.

Todos estos aspectos se ilustran modélicamente desde los primeros minutos de la película, donde el Maestro de Ceremonias del Kit Kat, impecablemente interpretado por Joel Gray, entona una canción de bienvenida que parece dirigida tanto al público del cabaret como al espectador de la película.

La aparición de este personaje a través de un espejo deformante -cuyo alto valor simbólico retomaremos al final de estas páginas- ilustra, desde el principio, su carácter en cierto modo fantasmagórico y ambiguo, ya que actúa como un narrador intradiegético que pertenece tanto al ámbito del «relato» como al de la «historia» (Génette 1989b): su función en la película, en efecto, es la de apostillar, presentar y participar en los números musicales y, salvo fugaces apariciones en otros espacios, sus intervenciones se limitan al ámbito cerrado del cabaret. Es muy significativo, además, que este personaje no aparezca individualizado con un nombre propio, sino que nos refiramos a él sencillamente como el «Maestro de Ceremonias», pues, igual 
que ocurre en la exquisita película de Max Ophuls La Ronda (1950), que cuenta también con un guía intradiegético, su razón de ser no consiste tanto en interactuar con las criaturas del microcosmos fílmico como en acompañarnos en la contemplación de sus peripecias.

A partir, por tanto, de que el Maestro de Ceremonias irrumpe con su canción de bienvenida («Wilkommen»), empieza Fosse a utilizar la técnica del montaje paralelo que nos muestra -no necesariamente en coexistencia simultánea- situaciones que se desarrollan tanto en el interior como en el exterior del local. Vemos, así, de forma intercalada cómo llega a Berlín el personaje de Brian (convirtiéndose en un tercer destinatario de esa canción de bienvenida), trasunto del narrador autobiográfico del libro de Isherwood ${ }^{4}$, al tiempo que el Maestro de Ceremonias nos presenta a los integrantes del espectáculo.

En un nuevo efecto especular, el personaje de Brian comparte con el innominado Maestro de Ceremonias desde esta inicial secuencia su condición de observador crítico de la enloquecida Alemania de entreguerras. Sin embargo, la omnisciencia de uno y otro no es la misma, pues Brian sí se incorpora desde el momento en el que conoce a Sally Bowles -protagonista indiscutible de la película y estrella del Kit Kat- al ámbito de la «historia», simultaneando así una doble condición de observador y personaje que inevitablemente lastra una objetividad que sí mantiene intacta el Maestro de Ceremonias, cuya crucial función en la película es, como apuntábamos más arriba, claramente metafórica (Mizejekski 1992: 180): su mirada es, a la vez, la nuestra, la del espectador conocedor de los hechos históricos y parte de una perspectiva privilegiada que le permite percibir la verdadera situación de Berlín y del pequeño cabaret que la refleja.

Volviendo al número musical que abre la película, en la letra de «Wilkommen» se nos presenta una oposición -que, como iremos viendo a medida que avance la película, es solo aparente- entre el Kit Kat y el mundo exterior cuando el Maestro de Ceremonias, con una voz y una mirada tan irónicas como sus propias palabras, declara al público que «afuera hace viento», en una evidente mención metafórica al revuelto contexto político berlinés, mientras en obvia dilogía sexual, dentro del cabaret todo es «caliente», y tanto las chicas (muchas de ellas travestis) y la desafinada orquesta son «hermosas».

A pesar, pues, de esa ilusoria diferencia entre el «frío» exterior y el «calor interior», el espacio del Kit Kat se convierte desde el inicio de la película en ese microrreflejo de una realidad nada complaciente, en la medida en que el propio Fosse

empezaba a percibir que en los bajos fondos del mundo del espectáculo se escondía una metáfora, según la cual toda la existencia era vulgar y corrupta. Pareció llegar a la conclusión de que la vida era inocencia corrompida, el garito de strip-tease exprimido a su mínima esencia, y eso tanto en la vida como sobre la escena. (Gottfried 2006: 293)

${ }^{4}$ Es interesante mencionar aquí la novela de Arthur R. G. Solmssen (1982) Una princesa en Berlín, que reconstruye también este confuso período de la historia alemana desde la perspectiva del visitante extranjero, convertido, igual que nuestro Brian, en espectador y personaje del argumento. 
Por todo lo que venimos diciendo, no sorprende que esta primera secuencia de la película, que constituye, a la vez, el primer número musical, tenga una puesta en escena eminentemente pictórica, que persigue un efecto expresionista, con colores excesivos, gestos histriónicos y un vestuario completamente alejado del glamour habitual en el musical hollywoodiense. Como indica Gottfried (2006: 200), Fosse se inspiró sobre todo en la película Moulin Rouge, de John Huston, «en lo que se refiere a color y composición», aunque no es difícil encontrar influencias pictóricas como la del célebre Retrato de la periodista Silvia von Harden, de Otto Diz, que aparece prácticamente calcado en una de las andróginas espectadoras del cabaret:
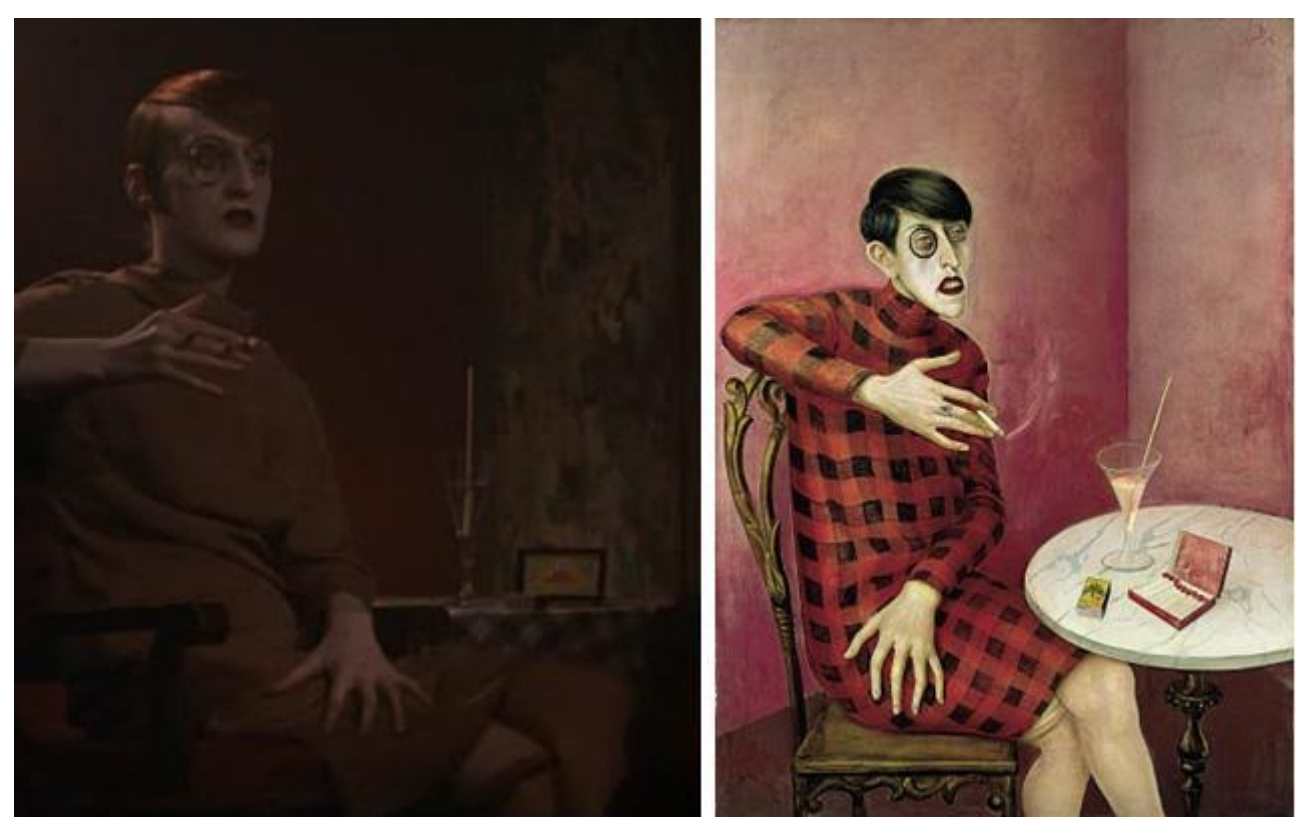

Fig. 1. Fotograma de Cabaret / Otto Dix: Retrato de la periodista Silvia von Harden

Nada más apropiado para ilustrar la ambigua naturaleza del público que este personaje inspirado en el cuadro de Dix, ya que es justamente la pasividad de los espectadores lo que los convierte en trasunto de la sociedad alemana, insistiendo de esta manera en la estructura en abismo que venimos mencionando:

La característica imagen recurrente de ese cabaret, exquisitamente grotesco, consistía en los rostros inmóviles de los alemanes entre el público, las caras de los burgueses observando indolentemente las actuaciones de contorsionistas y travestis, e incluso escenas de azotes, como si fueran un entretenimiento. Realmente el espectáculo que se ofrecía en ese cabaret no era malvado, la maldad radicaba en los rostros del público, indiferentes, insensibles, desalmados e impasibles. Las únicas expresiones de júbilo que Fosse permitió a los miembros del público fueron ante el espectáculo de unas mujeres que luchaban en el barro. 
No era demasiado necesario subrayar más el paralelismo entre esos observadores y la Alemania de 1931, sentada impasible ante el espectáculo nazi. (Gottfriet 2006: 297)

En efecto, ya avanzando en la película -donde asistimos progresivamente a las relaciones entre los personajes, especialmente a sendos romances entre Brian y Sally Bowles, de la que nos ocuparemos más adelante, y entre Fritz y una adinerada joven de origen judío, muy importante para ilustrar la xenofobia nazi-, vamos observando cómo tanto los números musicales como las coreografías se vuelven cada vez más oscuros y violentos, aunque siempre desde esa óptica expresionista y casi esperpéntica de la que venimos hablando.

Detengámonos en algunos de estos números musicales. El primero de ellos es el que muestra una pelea coreografiada en la que los bailarines se comportan casi como si fueran marionetas al son de una música que parece proceder de una caja mecánica. Dicha pelea se yuxtapone a una paliza que unos jóvenes neonazis propinan al dueño del Kit Kat, que los había expulsado del local en una secuencia anterior. El contraste entre el tono infantil y desenfadado de la coreografía con la violencia de la acción paralela no puede ser más llamativo y provoca en el espectador, ya desde el principio de la película, una incómoda sensación de que, efectivamente, el Kit Kat no constituye ese supuesto refugio contra el violento «viento» exterior que se le prometía al iniciar la película.

El mismo efecto produce la lucha entre mujeres en el barro, recuerdo de las que el propio Fosse contempló en sus periplos por los bajos fondos de distintas ciudades alemanas y cuya inequívoca relación con el mundo real aparece eficazmente subrayada por el Maestro de Ceremonias, quien, en un guiño tristemente proléptico, se pinta con el mismo barro del escenario un bigote que lo convierte en la contrafactura, a un tiempo risible y espeluznante, del propio Hitler, en un intento de ridiculización de la figura autoritaria que enlaza con la estética degradante anunciada en el número de bienvenida y que podemos relacionar con parodias similares en películas como El gran dictador (1940), de Charles Chaplin, o Ser o no ser (1942), de Ernst Lubitsch.

Asimismo, llama también la atención el número titulado «Tiller girls», en el que observamos también la ridícula danza de un nada marcial ejército compuesto por mujeres semidesnudas a la orden de un carcajeante Maestro de Ceremonias, que se yuxtapone esta vez a una de las más terribles escenas de la película, aquella en la que un grupo de nazis asesina al perro de la joven judía mencionada más arriba.

Este hábil manejo del lenguaje cinematográfico explica que Cabaret se encuentre por derecho propio entre las películas que protagonizaron la innovación del cine americano de los años setenta: pensemos, por ejemplo, que un procedimiento similar utilizó Francis Ford Coppola en la primera entrega de El Padrino, estrenada justamente el mismo año, y en la que observamos tanto la técnica del montaje simultáneo - pensemos en la secuencia del bautizo, entrelazado con una matanza entre bandas- como la expresión de la violencia de un modo novedoso e impactante, ya que la inolvidable imagen de la cabeza del caballo muerto en la cama de uno de los personajes posee sin duda el mismo efecto aterrador que la del perro de la secuencia de Cabaret que ahora nos ocupa.

Avanzada la película, los personajes salen del entorno de Berlín y el cabaret guiados por un ambiguo personaje, un aristócrata de moral difusa que de algún 
modo compra la compañía de nuestra pareja protagonista y a través del cual Fosse sugiere la muy moderna idea de la homosexualidad y el triángulo amoroso. De camino hacia su lujosa residencia de verano, hacen una parada en un restaurante al aire libre donde un joven empieza a cantar «Tomorrow belongs to me», canción que inicialmente parece ser un entusiasta canto a la primavera y al trabajo, con motivos fácilmente reconocibles como la abeja y el ciervo -símbolos respectivamente de la «laboriosidad, creación y riqueza» y la «renovación y el crecimiento» (Cirlot 1984: 49 y 128) - y acaba convirtiéndose en una apología de la cosmovisión nazi que envuelve a los clientes hasta convertirlos en exaltados intérpretes de un himno colectivo. Destaca esta secuencia por la inteligentísima utilización que hace Bob Fosse de los recursos del lenguaje cinematográfico, a través de eficaces movimientos de cámara que nos van ofreciendo progresivamente información sobre los personajes y la situación que protagonizan: empieza con un primer plano del solista para ir avanzando hasta un plano medio en el que se observa su uniforme de miembro de las juventudes nazis y continúa hasta un plano general que nos muestra, sin necesidad de palabra alguna, cómo el mensaje de la canción se ha extendido hacia un público - una sociedad- que canta enardecido mientras hace el inequívoco saludo fascista. La iconografía de esta secuencia nos remite claramente a la película de propaganda nazi El triunfo de la voluntad, de Lenie Riffenstahl, como bien apunta Mizejevski (1992: 176) cuando se refiere al proceso que convierte al público en parte del espectáculo. Solo dos espectadores se muestran alarmados ante lo que allí está sucediendo: un anciano que no participa de la canción y que muestra una visible cara de desagrado y preocupación y, naturalmente, Brian, cuya función como observador imparcial quedó establecida desde el principio de la película.

No se puede, en efecto, decir más con tan pocos elementos: la evolución de la canción y de la gestualidad de sus intérpretes es una clara muestra de la imparable ascensión del nazismo, sin innecesarios subrayados ni más comentarios que el que Brian interpela a su ingenuo anfitrión alemán cuando retóricamente le pregunta si de veras cree que va a ser posible poner freno a ese movimiento. Termina la secuencia con un fugaz primer plano del Maestro de Ceremonias que refuerza, una vez más, con su sonrisa maléfica y omnisciente, la gravedad de lo que acabamos de contemplar en uno de los pocos momentos en los que aparece fuera del entorno del Kit Kat (el otro es, tampoco por casualidad, cuando Sally conoce al aristócrata y el Maestro de Ceremonias avanza el contenido de la celebérrima canción «Money», cuya letra y situación en el argumento, dada la demonización de la comunidad judía a causa del dinero, son igualmente significativas).

«Tomorrow belongs to me», pese a ser uno de los números musicales de los que Fosse se arrepintió (Gottfried 2006: 285), tiene una gran importancia dentro de la película, porque refuerza la función diegética de la canción al sacarla fuera del entorno distorsionado del cabaret, lo que la convierte no ya en una premonitoria sensación sino en una realidad palpable ${ }^{5}$. Asimismo, no parece gratuito el hecho de que

5 Tal es, en fin, la fuerza de este himno que ha devenido en paradigma de la cosmovisión fascista, tal y como se muestra en la ácida serie Orange is the new black (Kenji Lojan, 2013), concretamen- 
Sally no aparezca en esta secuencia, lo que refuerza su inconsciencia y su alejamiento de la realidad social en su monotemático afán por lograr el éxito profesional.

Los acontecimientos se precipitan a partir de esta secuencia, al tiempo que se enrarecen tanto la relación entre Sally y Brian como el ambiente berlinés. En efecto, la relación amorosa entre los protagonistas dista mucho de reproducir el habitual esquema de la comedia romántica $\mathrm{y}$, tanto por la proclamada libertad sexual de la cantante, ampliamente analizada por Mizejevski (1992), como por las insalvables diferencias entre ambos, está inevitablemente condenada al fracaso.

De este modo, Fosse ahuyenta rotundamente toda similitud entre su propuesta fílmica y un musical hollywoodiense al uso. Para ello, empieza, en primer lugar, renunciando al happy ending (Castells 2005: 167-68) no solo a través de la lúcida negativa por parte de Sally a creer en una improbable felicidad conyugal (anunciada prolépticamente, de hecho, en la icónica canción «Mein Herr», creación original para nuestra película) sino también incorporando al argumento el escabroso y nada habitual tema del aborto.

Por otra parte -y es lo que nos interesa ahora-, se recrudecen los números musicales, cuya violencia aumenta intensificando su condición de correlatos distorsionados pero elocuentes del creciente clima de agitación prebélica. Es ahora, por ejemplo, cuando se desarrolla la secuencia anteriormente mencionada del asesinato del perro de la mujer judía y cuando Brian, abandonando su característica flema de observador desapasionado, se enzarza en una pelea con unos jóvenes nazis tras la cual abandona definitivamente la capital berlinesa y todo lo que de ella se refleja en el espejo del cabaret.

Llegamos, así, al momento culminante de Cabaret: aquel en el que una insuperable Liza Minelli ya convertida en mito interpreta con valentía, pasión y absoluta verdad el tema que da título a la película. Tras una de las más bellas despedidas amorosas de la historia del cine y después de dejar a Brian en la misma estación ferroviaria en la que lo vimos por primera vez, el círculo argumental se cierra y solo queda, imponente, nuestra protagonista, entonando, apenas iluminada por unos focos que ensalzan su figura señera, un canto a la libertad y a la independencia, a una vida berlinesa que -nosotros sí lo sabemos- tardará poco en desintegrarse.

Detengámonos brevemente en algunos fragmentos del tema que da título y fama a la película:

What good is sitting, all alone in your room?

come hear the music play!

life is a cabaret, old chum!

come to the cabaret!

[...]

I used to have this girlfriend known as Elsie

with whom I shared for sordid rooms in Chelsea

te en el capítulo quinto de la cuarta temporada, donde la canción suena para subrayar la inminente gestación de una organización xenófoba dentro de la prisión. 
she wasn't what you'd call a blushing flower

as a matter of fact she rented by the hour.

The day she died the neighbors came to snicker

well, that is what comes from too much pills and liquor.

But when I saw her laid out like a queen

she was the happiest corpse I'd ever seen.

I think of Elsie till this very day

I remember how she turned to me and said:

what good is sitting all alone in your room?

come hear the music play

life is a cabaret, old chum!

come to the cabaret!

And as for me, and as for me,

I made my mind up back in Chelsea

when I go, I'm going like Elsie.

Start by admitting from cradle to tomb

it isn't that long stay

life is a cabaret, old chum!

it's only a cabaret, old chum!

and I love the cabaret! ${ }^{6}$

Dos tópicos clásicos en nuestra tradición cultural -el carpe diem y el theatrum mundi-se aúnan en esta exaltación del cabaret como espacio de libertad, claramente ilustrado a través de la mención de la historia de Elsie, fallecida después de una existencia entregada a los placeres y cuya presencia en la canción constituye una nueva estructura en abismo que parece justificar la rotunda apuesta de nuestra Sally por sumergirse en el gozoso microcosmos del escenario. De forma muy significativa y eficaz, en las tomas del último número musical de la cantante, el público aparece de espaldas, apenas en escorzo, sin que se nos muestren, como sí se hizo al principio, ni su gestualidad ni sus rostros. Fosse consigue, así, aislar a Sally de lo que está ocurriendo y realzar el contraste con los planos finales de la película.

Porque, en efecto, lo que en un principio podría resultar un final emocionante y vitalista acaba por adquirir un tono agridulce a través de la última y definitiva aparición del Maestro de Ceremonias, que reproduce fragmentos de la canción de bienvenida, convertida ahora en despedida, y cierra la película en una desasosegante estructura circular.

${ }^{6}$ ¿De qué sirve quedarte a solas en tu habitación?/ Ven y oye cómo suena la música./ La vida es un cabaret, viejo amigo,/ ven al cabaret./ [...] Yo tuve una amiga llamada Elsie / con la que compartí sórdidos pisos en Chelsey./ No era precisamente una flor ruborosa,/ de hecho, se alquilaba por horas.../ El día que murió los vecinos fueron a burlarse:/ «eso pasa por demasiadas pastillas y licor»./ Pero cuando la vi yaciendo como una reina.../ era el cadáver más feliz que he visto nunca./ Pienso en Elsie casi cada día / y recuerdo cómo se volvía hacia mí y decía:/ ¿De qué sirve quedarte a solas en tu habitación?/ Ven y oye cómo suena la música./ La vida es un cabaret, viejo amigo,/ ven al cabaret./Y en cuanto a mí,/ tomé mi decisión en Chelsey / y cuando me vaya, / lo haré como Elsie. / Empieza admitiendo que de la cuna a la sepultura / el camino no es muy largo./ La vida es un cabaret, viejo amigo,/ Es solo un cabaret, viejo amigo / Y yo amo el cabaret. (La traducción es nuestra, directamente extraída de la película). 
Pero el Kit Kat ya no es el mismo: las notas de la orquesta suenan ahora desafinadas y a ritmo lento, como en progresiva agonía, al modo de un antiguo vinilo reproducido a pocas revoluciones, mientras se proyectan, ralentizadas, imágenes anteriores de la película, recuerdos de un ambiente lúdico y desprejuiciado definitivamente en extinción. Este inquietante número final, puesto en relación con la canción anterior, revela claramente, pues, que Sally, inconsciente en su hedonismo autodestructivo, parece estar optando por convertirse, como su amiga, en un cadáver feliz, en ese Berlín que no tardará en convertirse en un inmenso cementerio en el que yacen la diversión y la libertad.

Y esto es así porque Cabaret termina con un descorazonador travelling en el que la cámara -la misma que erigía Isherwood como instrumento para su retrato- nos ofrece, superpuesta a los títulos de crédito, la imagen de un público muy diferente del que vimos en la primera secuencia de la película. Quienes contemplan la vehemente actuación de Sally -y es justo ahora cuando, para acentuar este crudo final, Fosse ha decidido mostrárnoslos- no son ahora personajes anónimos que disfrutan de unas horas de evasión: son militares nazis que observan con sus rostros amenazantes un espectáculo que no parece emocionarles en modo alguno. En este travelling final, además, la música de la orquesta ha sido sustituida por un redoble de tambor de tono inequívocamente militar que se detiene justo cuando Fosse decide congelar la imagen y acabar la película con un primer plano de la esvástica que exhibe en su uniforme uno de los militares del público.

«Life is a cabaret», proclama Sally desde su enajenada y sesgada visión del mundo: «War is a cabaret», podríamos corear nosotros, desde el momento en que vemos que los nazis, y lo que su presencia implica, ya están dentro del Kit Kat.

El cabaret ya no es refugio. Tampoco es, ni siquiera, reflejo, porque el horror y la guerra están ya en su interior, dominándolo todo: primero, las coreografías y números musicales y, finalmente, ya de forma irreversible, al propio público, que ha pasado, como vimos también en la secuencia de la canción «Tomorrow belongs to me», de ser espectador pasivo a protagonista de una realidad horripilante y ominosa. El «viento» exterior del que prometía protegernos el Maestro de Ceremonias en el número musical «Wilkommen» se ha colado ya dentro del Kit Kat y ya no hay canción ni baile ni juegos eróticos que puedan hacernos olvidarlo.

El último plano de la película -la amenazadora esvástica del uniforme de un espectador-, como la primera aparición del Maestro de Ceremonias, emerge también de un espejo deformante, exhibiéndose ante nosotros con la paradójica veracidad del esperpento. Afirmaba Max Estrella en la célebre escena XII de Luces de Bohemia que «el sentido trágico de la vida [...] solo puede darse con una estética sistemáticamente deformada» (Valle-Inclán 2000: 162-163). Los espejos cóncavos con los que abre y cierra Bob Fosse su película son los mismos que, desde el callejón del Gato, utilizó Valle-Inclán para mostrarnos, bajo la máscara del esperpento, la más nítida imagen de una época.

\section{Referencias bibliográficas}

CASTELlS, Isabel (2005): « ¿Y comieron perdices? Amores adúlteros vs. comedia romántica». Latente 3: 161-178. 
Cousins, Mark (2005): Historia del cine. Barcelona: Blume.

CIRLOT, Juan Eduardo (1984): Diccionario de símbolos. Barcelona: Labor.

DÄLLENBACH, Lucien (1991): El relato especular. Madrid: Visor.

GENETTE, Gérard (1989a): Palimpsestos. Madrid: Taurus.

- (1989b): Figuras III. Barcelona: Lumen.

GotTFrIED, Martin (2006): Bob Fosse. Vida y muerte. Barcelona: Alba.

ISHERWOOD, Cristopher (2014): Adiós a Berlín. Barcelona: Acantilado.

MizeJewsKi, Linda (1992): Divine Decadence: Fascism, Female Spectacle, and the Makings of Sally Bowles. Princeton, New Jersey: Princeton University Press.

RoDA, Arlene (1994): «Cabaret: utilizing the film medium to create a unique adaptation». Literature Film Quaterly 22-1: 36.

SÁnCHEZ NoriegA, José Luis (2000): De la literatura al cine. Teoría y análisis de la adaptación. Barcelona: Paidós.

- (2006): Historia del cine. Teoría y géneros cinematográficos, fotografía y televisión. Madrid: Alianza Editorial.

Solmssen, Arthur R. G. (1982): Una princesa en Berlín. Barcelona: Tusquets.

VALLE-INCLÁN, Ramón María (2000): Luces de bohemia. Madrid: Espasa-Calpe.

WHITE, Hayden (2011): La ficción de la narrativa. Ensayos sobre historia, literatura y teoría. 1957-2007. Buenos Aires: Eterna Cadencia.

\section{Filmografía}

Chaplin, Charles (dir.) (1972): El gran dictador. Estados Unidos: United Artists. 1940. [Película]

CopPOLA, Francis Ford (dir.) (1972): El padrino. Estados Unidos: Paramount Pictures / A. S. Ruddy Production. [Película]

CORNELIUS, Henry (dir.) (1955): I am a camera. Reino Unido: Romulus Films. [Película]

Fosse, Bob (dir.) (1972): Cabaret. Estados Unidos: Allied Pictures / ABC Pictures. [Película]

LOJAN, Kenji (dir.) (2013-2016): Orange is the new black. Estados Unidos: Netflix. [Serie de televisión]

LuBITSCH, Ernst (dir.) (1942): Ser o no ser. Estados Unidos: Romaine Film / Alexander Korda. [Película]

OPHÜls, Max (dir.) (1950): La ronda. Francia: La Ronde. [Película]

RIEFENSTAHL, Lenie (dir.) (1935): El triunfo de la voluntad. Alemania: Reichsparteitagsfilm. [Documental]

SolOWAY, Jill (dir.) (2014): Transparent. Estados Unidos: Amazon Studios / Pictures in a Row. [Serie de televisión] 